\title{
Application of Time-domain Average and Wavelet on Fault Diagnosis in Gear
}

\author{
KANG Haiying $^{1, a}$, LIU Guangsheng ${ }^{1, a}$, HE Yaoxin ${ }^{1, a}$, JIA Changzhi ${ }^{1, a}$ \\ ${ }^{1}$ Shijiazhuang Mechanical Engineering College, Shijiazhuang 050003, China) \\ aemail:hykangqi@163.com
}

Keywords: time domain average; wavelet; gear; fault diagnosis.

Abstract. Time-domain average is a common method of extracting cycle heft that we are interested in from complex cycle signals that get along with noise. When it was put into practice, because of cycle truncation error, it can't gain satisfactory effect. The fault vibration signals of the gear were measured in this paper and a new arithmetic of time-field average was put forward which can settle the problem of cycle truncation error affect the result of averaging in substance, and then the outcomes was decomposed and redecomposed with wavelet. The result shows that during processing the fault signals of the gear the time-average method can eliminate the influence of the background noise and can acquire obvious efforts.

The each signals which reflecting the circulation state of the gearbox is repeated with periodicity following the movement of gearbox, and the frequency is the multiple circumgyrate frequency of the machinery. But there is more noise disturbs in fact engineering application. In the frequency spectrum, the cycle components can be annihilated by background noise. So, how to improve the signal-to-noise of the signals is the basic question.

The time-domain average is one in common use method; the cycle component which is interested is distilled from the complex cycle signal which is mixed with noise. The signals ponderance which is irrespective with the specified frequency, such as the gyro frequency of shaft axle, is removed. The noise and the cycle signals which is irrespective can remove. The cycle signal which is correlation with the specified frequency is distilled. The signal-to-noise of signals is enhanced with the time-domain average method. But in fact, the effection is unsatisfied because the cycle truncation error [1-2].

It is dissimilarity as the usual signals collection, in the paper, not only the analysis signal is collected, but also the timing pulse of rotor is collected. So the origination of each signal segments can be locked.

\section{The theory of time-domain Average}

The $\mathrm{x}(\mathrm{t})$ is the mechanism signals which is generated by the rotary machine circulation. The corresponding discrete signals is $x_{n}=\mathrm{x}(\mathrm{n} \Delta)$, the $\Delta$ is sampling interval. The corresponding cycle signal is distilled according to gyro frequency $f_{0}$. The $x_{n}$ is dipartite into $\mathrm{P}$ segments. The cycle of each segment is $T=1 / f$. The check the number of sampling in each segments is equality and the number is $\mathrm{N}$. the time-domain average can denotation as,

$$
\overline{x_{n}}=\frac{1}{p} \sum_{p=0}^{p-1} x_{n+p N}
$$

The formula (1) is analyzed with $\mathrm{Z}$ commutation,

$$
\overline{x(z)}=\frac{1}{p} \sum_{p=0}^{p-1} z\left[x_{n+p N}\right]
$$

It can be obtained with the time-shife characteristic of $\mathrm{Z}$ commutation, 


$$
\overline{x(z)}=\frac{1}{p} x(z) \sum_{p=0}^{p-1} z^{p N}
$$

And then let $z=e^{j 2 \pi f \Delta}$, the frequency correspond function of the time-domain average can be obtained,

$$
H(f)=\frac{1-e^{j 2 \pi f \Delta p N}}{p\left(1-e^{j 2 \pi f \Delta N}\right)}
$$

Because the $\Delta N=T=\frac{1}{f_{0}}$, the $\mathrm{H}$ (f) can be obtained,

$$
H(f)=\frac{e^{j \pi p \frac{f}{f_{0}}\left(e^{-j \pi p \frac{f}{f_{0}}}-e^{j \pi p \frac{f}{f_{0}}}\right)}}{p e^{j \pi \frac{f}{f_{0}}}\left(e^{-j \pi \frac{f}{f_{0}}}-e^{j \pi \frac{f}{f_{0}}}\right)}
$$

At last, the amplitude frequency character and the phase frequency character are obtained,

$$
|H(f)|=\frac{1}{p}\left|\frac{\sin \left(p \pi \frac{f}{f_{0}}\right)}{\sin \left(\pi \frac{f}{f_{0}}\right)}\right| \quad \phi(f)=\frac{\pi(p-1) f}{f_{0}}
$$

The amplitude frequency response characteristics and the phase-frequency response characteristic of the time-domain average is composed with a series of the band-pass optical filter and side band which is distributed as isometric, it is the comb filter. The phase-frequency characteristic and the frequency is linearity connection. The center frequency of band-pass optical filter is the multiple of gyro frequency $f_{0}$. When the average time $\mathrm{P}$ is larger, the pass band breadth is becoming narrow and the peak value of side band is attenuation quickly. So the cycle signal which is correlative with the gyro frequency $f_{0}$ is distilled with the time-domain average. The noise and noncorrelation signals are removed. The signal which is interested is distilled with the propriety $f_{0}$.

\section{Implementation of the algorithm}

The $\Delta t$ is the distant in sampling sequence $\mathrm{x}(\mathrm{n}), n=1,2,3, \mathrm{~L} N_{1}$. The cycle of ponderance which is interested is $\mathrm{T}$. The time-domain average new arithmetic is ${ }^{[4-6]}$,

$$
y(m)=\frac{1}{p} \sum_{k=0}^{p-1} x(m-k l) \mathrm{L} \quad\left(m=N_{1}-L+M, N_{1}-L+2 M, \mathrm{~L}, N\right)
$$

Where, the $y(\mathrm{~m})$ is the new sequence which is obtained after average. The $\mathrm{p}$ is the average segment. The $\mathrm{L}$ is the approximately round numbers of average segment. The $\mathrm{M}$ is resampling interval in each average segment length. $M=\frac{T}{\Delta t}=\frac{f_{s}}{f}$, the $f_{s}$ is sampling frequency. The $f$ is frequency of cycle ponderance which is interested.

Now the question is how to select the average segment data p. In one time sampling sequence, we can't judge how many cycle which is interested. In order to solve the question, the photo electricity sensor is installed in the select axes. The timing pulse and sampling pulse are exported in the same time. The sampling pulse sequence which is corresponding the timing pulse is sampling, so the consistency of both of them is assured. 


\section{The arithmetic of wavelet}

The wavelet analysis method is one kind of time-frequency localize analysis method which the window size is fixation (window area), but the figure can changeable. It is superiority than Fourier transform. It has good localize character both in time-domain and in frequency-domain.

The Matlab arithmetic is one method which the signals is analyzed with a group of band-pass optical filter, so the components in differ frequency channels are obtained through decomposing the signals. Firstly, the signals are decomposed into the low-frequency and high-frequency components. Secondly, the low-frequency signals which is obtained last time decomposition is decomposed into low-frequency and high-frequency components in each decomposition. So the data is halving in each decomposition and the sampling time is increase double. After the signal is decomposed, it can be reconfigurated with the restructing algorithm, the length and sampling interval of original signals are resumed. The restructing algorithm is as follows ${ }^{[7]}$,

$$
c_{k}^{j}=\sum_{n} c_{n}^{j+1} h_{k-2 n}+\sum_{n} d_{n}^{j+1} g_{k-2 n} \quad(k=0,1,2, \mathrm{~L}, N-1)
$$

The Fig 1 is the decompose course of the multi-differentiate analysis.

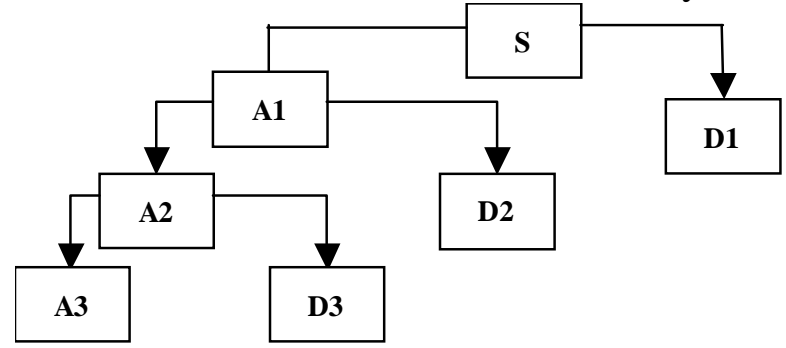

Fig 1 the decompose tree of multi-differentiate analysis (three floor)

In the Fig 1, the $\mathrm{S}$ is original signals, the $\mathrm{Ai}$ is the low-frequency components of the I floor with multi-differentiate analysis, the $\mathrm{Di}$ is the high-frequency components of the I floor with multi-differentiate analysis. The original signals can be expressed with, $\mathrm{S}=\mathrm{A} 3+\mathrm{D} 3+\mathrm{D} 2+\mathrm{D} 1$ 。

\section{The example of gearbox diagnosis}

The examination is certificated in one kinds of single-stage gearbox. In the system, the input axis is driven by a speed-regulating electric motor; and the magnetic powder load is driven by the output axis. The number of teeth in drive gear is $Z 1=30$, the number of teeth in driven gear is $Z 2=50$. The model of rolling bearing in input is 206. The gear tooth of initiative gear is burnished simulating the fault of wear. The vibration signals and rotate speed signals are sampled at constant time increments in time-domain, the frequency of sampling is $16384 \mathrm{~Hz}$; the sampling time is $2 \mathrm{~s}$. The mesh frequency of gear is $289 \mathrm{~Hz}$. The vibration signals with acceleration sensor and the tinning pulse signals with photoenectricity sensor are delivered the B\&K3560 multi-analysis meter, and then the signals in time-domain is obtained.

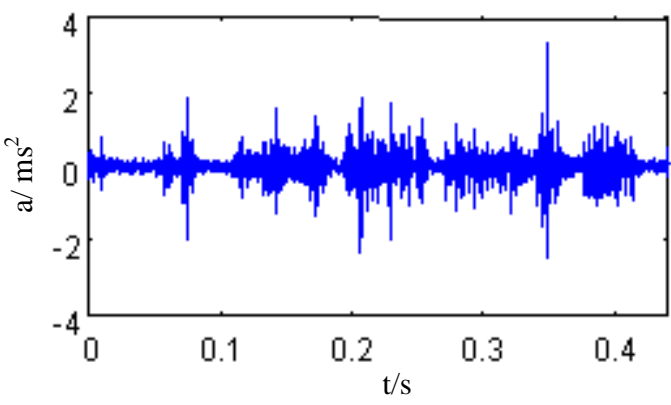

Fig. 2 The original vibration signals

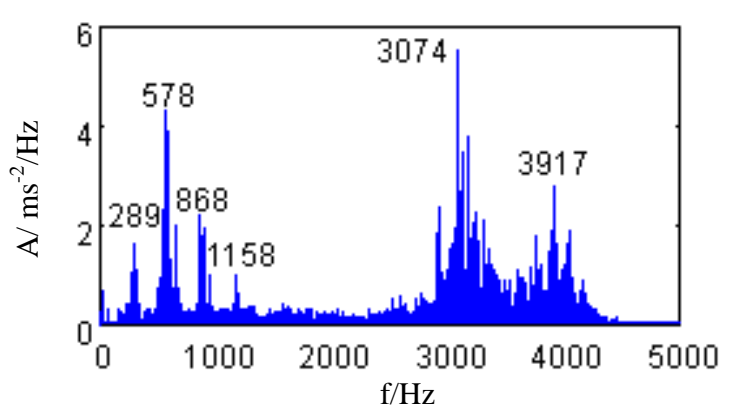

Fig.3 The frequency spectrum of original vibration signals

The amplitude of vibration is large because there is background noise in the original vibration signals (Fig.2). 
In the frequency spectrum of original vibration signals (Fig.3), there are included both the useful mesh information of gear and the infection of noise. The energy amplitude is larger. The low-frequency segment is reflected the mesh complexion of gear.

The amplitude of vibration signals is weaken obvilus after the original vibration signals are disposed with time-domain average. Much useless information, such as stochastic noise and comparison noise, is removed.

It can be seen from the frequency spectrum of vibration signals after time-domain average (Fig.5), the energy amplitude after time-domain average is smaller than the original vibration signals. The amplitude of frequency spectrum in low-frequency segment is corresponded with the diversity diploid mesh frequency of the gear.
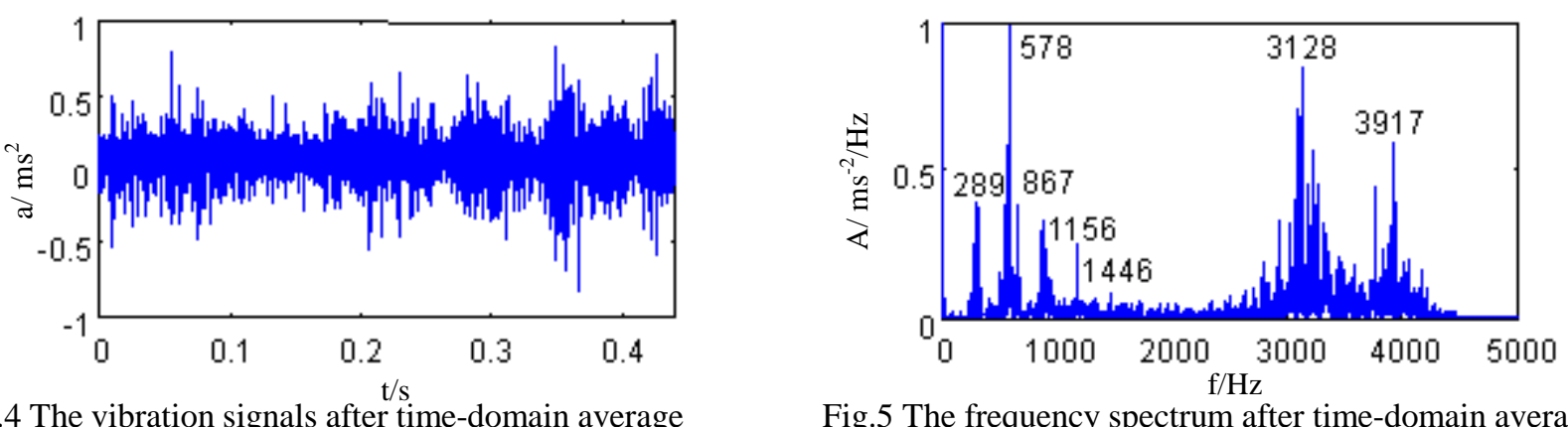

Fig.4 The vibration signals after time-domain average

Fig.5 The frequency spectrum after time-domain average

The vibration signals after time-domain average are analyzed with wavelet decomposition and reconfiguration, the useful information in low-frequency is distilled, and then the signal is analyzed with frequency spectrum (Fig.6). It can be seen that the high frequency component is filtrated with wavelet. There is only the low frequency mesh frequency of gear $289 \mathrm{~Hz}$, and the double mesh frequency $578 \mathrm{~Hz}$, and the triple and fourfold mesh frequency 867 and $1156 \mathrm{~Hz}$.

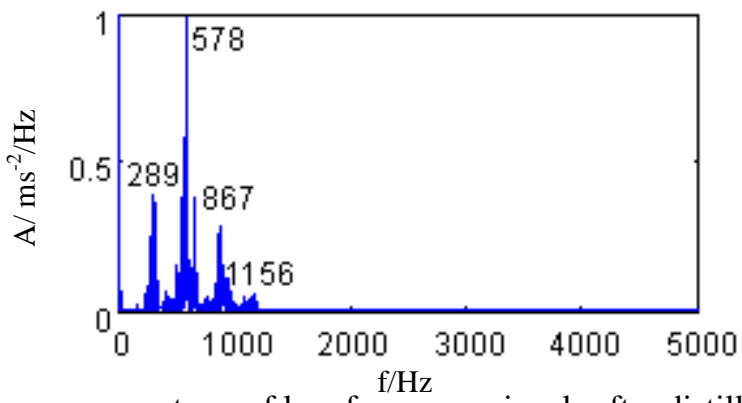

Fig.6 The frequency spectrum of low-frequency signals after distilling with wavelet

\section{Conclusions}

1) The list is additive and averaged which is according to same length in time-domain average old arithmetic. But in the new algorithmic, the timing pluse is cooperated to disposed the complexity vibration signals. The attenuation of cycle ponderance, which is generated by cycle truncation error, is overcomed. The effection is more obvious. The difference between the new and old algorithmic is visible. There is jumping-off error of each segment in old algorithmic and the error can be cumulated. But in new algorithmic, the jumping-off error of each segment is less than half sampling interval.

2) The time-domain average technique is application into the fault diagnosis of gearbox, the background noise and other useless information can be filtrated effectively. The useful vibration signals can be distilled from the originals signals. The diagnosis example proved the validity of the method. 


\section{References}

[1] Zhang Xian-da, Bao Zheng. Analysis and disposal of nonstationary signals [M]. National defense industry publishing company, 1998

[2] Wu Wen-tao, Lin Jing, Han Shao-bo. Time domain averaging based on nonintegral delay compensation $[\mathrm{J}]$. Vibration engineering transaction, 2009, 22(5):527-532

[3] LU Wen-xiang, Du Run-sheng. Signals analysis of mechanical engineering test information [M], Huazhong science and technology college publishing company, 1999

[4] Liu Hong Xing. A new algorithm of time domain averaging journal of Vibration engineering, Vol 12, No 3, sep. 1999,1。

[5] Chen Xin-hua, Sun Chang-yu, Bao Xi-zhong. Time domain averaging base on phase compensatons[J]. Applied acoustics, 2011, 30(4):268-274

[6] Xiao Zhi-song, Tang Li-wei, Wang Hong. Application of time-damain average on fault diagnosis of planetary gearbox [J]. Journal of hebei university of technology, 2003, 32(6):99-102

[7] Hu Chang-hua, Zhang Jun-bo, Xia Jun. analysis and design of system based on MATLAB_—analysis of wavelet [M]. xian electron science and technology publishing company, 1999 